\title{
A Literature Review on the Influence of LGBT Curriculum on Medical Students' Attitude Towards LGBT Patients
}

\author{
Han Zhao* \\ Shanghai Lida University, China \\ *Corresponding author: Han Zhao, zhaohanhz322@163.com
}

\begin{abstract}
Background: Since 2014, the American Academy of Family Physicians has proposed that medical residents should gain more understanding about LGBT so that LGBT (lesbian, gay, bisexual, and transgender) patients can gain access to healthcare without bias. In order to fulfil this, appropriate educational content on LGBT is required. Methods: Ovid, PubMed, and Website of Science have been searched for literatures published in last 8 years, from 2013 to 2021 . Literatures on the attitude of medical students before and after their experience of curricula with LGBT content were included, whereas those that focused on addressing the gap of LGBT content in the curricula, the bias towards LGBT medical students, and the effect of LGBT curriculum on the knowledge aspect were excluded. Results: Ninety-three articles were found, among which only six literatures were included in this review. It has been found that after LGBT education, students are more prepared and their attitude towards LGBT patients when providing healthcare is more positive. Some studies have also pointed out that medical students would change their opinion in treating LGBT patients. Conclusion: This review shows that there is evidence of the influence of LGBT education on medical students' attitude toward LGBT patients although a wide gap still exists in this field.
\end{abstract}

Keywords: LGBT; Medical education; Attitude towards LGBT patients; Healthcare

Publication date: December 2021; Online publication: January 24, 2022

\section{Introduction}

According to Gate and Newport, the LGBT population is large in many nations. There was a $3.4 \%$ increase in the United States only in $2012^{[1]}$. Hence, LGBT patients are requiring better health services. Unlike general patients, as an invisible community, LGBT patients face greater pressure when they start seeking health services. Some patients have even complained that their healthcare is problematic and inadequate as soon as they seek assistance from the hospital ${ }^{[2]}$.

In addition, research has demonstrated that discrimination widely exists in the services provided for LGBT patients ${ }^{[2]}$. The behaviors of many clinicians such as denying medical treatment (e.g., gender affirmation surgery) and incorrect pronouns may cause huge barriers when LGBT patients seek health services. As a result of their negative experiences with healthcare providers, LGBT patients tend to keep their personal information, such as their sexual orientation and gender identity, a secret from clinicians. Consequently, not only the trust between healthcare providers and patients will be impaired, but the lack of information and communication may lead to misdiagnosis as well. Moreover, they are more likely to suffer from mental disorders, such as anxiety, depression, and even suicide. According to the Human Rights Campaign, $40 \%$ of the participants have attempted suicide, and the number of individuals who have suicidal thoughts have doubled. 
In view of all the above factors, healthcare professionals should provide care for LGBT patients in a more cautious manner. However, many studies have indicated that there is almost nothing about LGBT in health sciences curricula, and some medical students even regard it as an unnecessary part of conventional medicine. Although the new requirement of including LGBT content in the curricula has been implemented recently, there are still obvious misunderstandings about LGBT patients among medical students and professionals. Therefore, both medical students and professionals should expand their knowledge on LGBT and change their attitude towards LGBT patients so that they can provide better and more suitable health services. In this literature review, studies about the accession of existing LGBT curriculum and the effective teaching methods of LGBT, which can influence the attitude of medical students, have been identified, but those about LGBT students and those that only addressed the gap were not referred to.

\section{Methods}

The protocol of this review is guided by SALSA (search, appraisal, synthesis, and analysis) ${ }^{[3]}$. The aim of this review is to assess the present relevant LGBT education approach from the perspective of students' attitude. In order to do this, Ovid, PubMed, and Web of Science have been searched using specific keywords: *Transgender Person or *Homosexuality, Female or *Sexual and Gender Minorities or *Bisexuality or *Homosexuality or *Homosexuality, Male or *Transsexualism or *Students, Medical AND *Education, Medical, Continuing or *Education, Medical; the filters included language (English), date (published from 2013 to 2021), and only peer-reviewed research articles. Thereafter, in order to meet the requirement of the research question, the titles and abstracts were checked to exclude irrelevant ones. Among the 53 screened literatures, those that focus on addressing the knowledge gap of LGBT without specific curriculum, the bias towards LGBT medical students, and the effect of LGBT curriculum on the knowledge aspect were excluded. Finally, MERSQI was used to assess the risk of bias as well as the quality of the study, in which the score of the articles was between 10.5 and 12.5.

The above process is described using the PRISMA Flow Diagram as shown in Figure 1, and this review solely focuses on those existing curricula on LGBT preparedness and attitude adjustment. Six studies, which had assessed different education measures (lectures, student-led presentations, and online lessons) using quantitative analysis, were found. Table $\mathbf{1}$ is a description of the included literatures. However, as the study designs of these researches are not similar, meta-analysis cannot be applied.

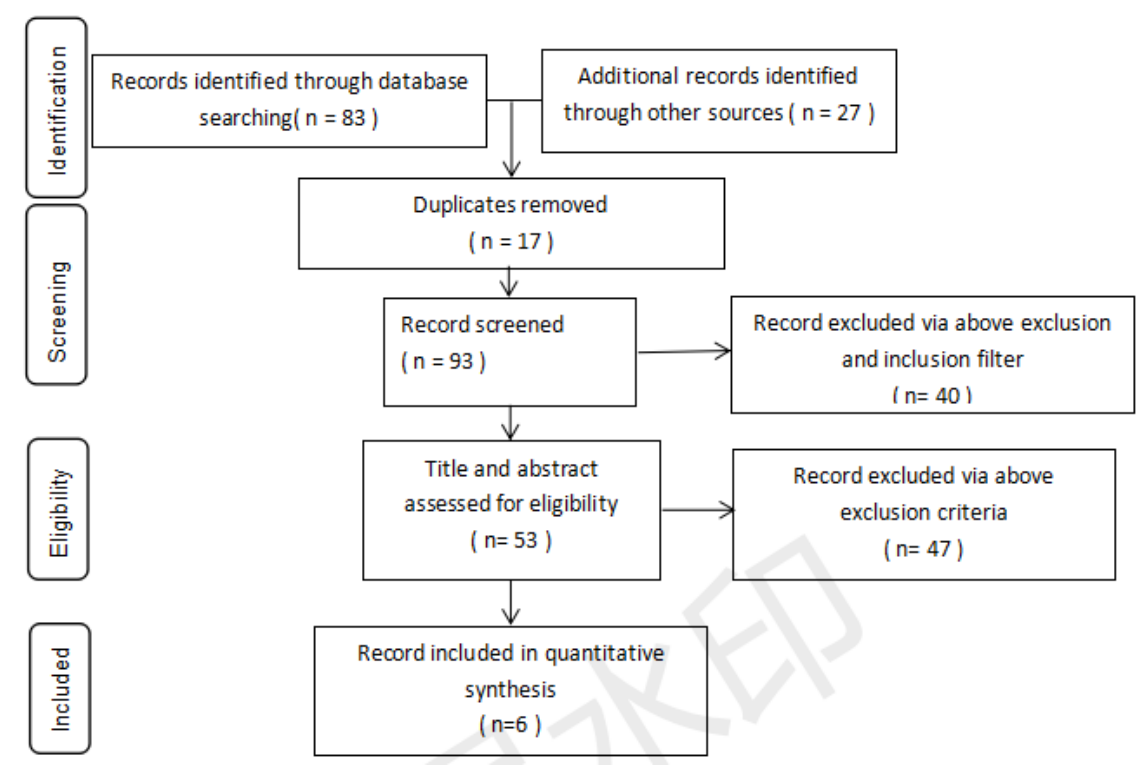

Figure 1. PRISMA Flow Diagram 
Table 1. Description of the included literatures

\begin{tabular}{|c|c|c|c|c|}
\hline Literature & Content and method & $\begin{array}{c}\text { Attitude/preparedness } \\
(P \text { value }) \\
\end{array}$ & State & Aim \\
\hline $\begin{array}{l}\text { Grosz, et } \\
\text { al. }{ }^{[4]}\end{array}$ & Presentation and terms & $P<0.001$ & USA & $\begin{array}{l}\text { To assess the knowledge and confidence in } \\
\text { providing care }\end{array}$ \\
\hline $\begin{array}{l}\text { Sawning, } \\
\text { et al. }{ }^{[5]}\end{array}$ & $\begin{array}{l}11 \quad \text { topics } \quad \text { about } \\
\text { communication and } \\
\text { practical skills }\end{array}$ & $P=0.037$ & USA & $\begin{array}{l}\text { To investigate attitude and knowledge } \\
\text { outcomes }\end{array}$ \\
\hline $\begin{array}{l}\text { Safer and } \\
\text { Pearce }\end{array}$ & $\begin{array}{l}\text { Treatment regimens and } \\
\text { requirements }\end{array}$ & $P<0.001$ & USA & $\begin{array}{l}\text { The attitude towards using hormones to treat } \\
\text { transgender individuals and related } \\
\text { experience }\end{array}$ \\
\hline $\begin{array}{l}\text { White, et } \\
\text { al. }{ }^{[7]}\end{array}$ & $\begin{array}{lr}16 \quad \text { topics } & \text { including } \\
\text { sexually } & \text { transmitted } \\
\text { infections } & \end{array}$ & $P<0.001$ & $\begin{array}{l}\text { USA / } \\
\text { Canada }\end{array}$ & $\begin{array}{l}\text { To characterize LGBT-related curricula and } \\
\text { evaluate their comfort and preparedness in } \\
\text { caring }\end{array}$ \\
\hline $\begin{array}{l}\text { Vishnu, et } \\
\text { al. }{ }^{[8]}\end{array}$ & Special terms & $P<0.01$ & UK & $\begin{array}{l}\text { Assess the use of terms and the attitude } \\
\text { towards LGBT }\end{array}$ \\
\hline $\begin{array}{l}\text { Kelley, et } \\
\text { al. }{ }^{[9]}\end{array}$ & Awareness and disparities & $0.001<P<0.57$ & USA & $\begin{array}{l}\text { To compare knowledge, attitudes, and beliefs } \\
\text { about LGBT before and after the intervention }\end{array}$ \\
\hline
\end{tabular}

\section{Results}

The results of this review are demonstrated below, which include the scope of research question and the description of the screened studies. All these small-scale studies are quantitative, with a limited number of data (from 20 to 166 participants) using self-reported questionnaires; P value was used to assess the change of attitude among the students. Most of these studies included medical students from the United States.

Grosz and other researchers conducted a study on student-led presentation by three second-year students and a fourth-year student at Case Western Reserve University School of Medicine in Cleveland, focusing on terms related to LGBT. The study reported that after the presentation, the students felt more comfortable in taking care of LGBT patients and had more confidence as well as preparedness $(P<0.001)$ [4].

Sawning and other researchers investigated the relationship between LGBT curriculum and medical students' attitude. Thirty-nine medical students (single group) were tested after an academic year of learning. From data analysis, there is evidence showing the relationship between LGBT education and students' attitude ${ }^{[5]}$.

A study led by Safer and Pearce involved non-randomized groups of students. Prior to transgender teaching, the students felt uncomfortable when taking care of LGBT patients and felt that LGBT health services should be apart from conventional medical services. However, with learning experience, $67 \%$ of second-year students reported that they feel more comfortable providing healthcare for LGBT patients ${ }^{[6]}$. White and other researchers observed several medical students from different schools in the U.S. and Canada and of different academic years, races, gender identities, and sexual orientations. After 5 hours of learning, data analysis demonstrated that the students' preparedness and feeling of comfort had improved significantly ${ }^{[7]}$.

Vishnu and other researchers carried out a study on 166 medical students in United Kingdom with different training conditions, gender identities, and sexual orientations. Eleven topics were tested in regard to students' attitude towards LGBT patients, among which six topics showed strong evidence for attitudechanging $(P<0.001)$ while 3 of them were larger than 0.1 (weak evidence). Compared with the score of 
specific LGBT terms, it is obvious that with training in LGBT terms, medical students' attitude will be affected ${ }^{[8]}$.

Kelley and other researchers observed 75 second year medical students in the U.S. before and after a 2-hour small-group experience. The results showed that there were significant changes in the responses for 4 out of 16 items in the questionnaire $(P \leq 0.01)$, with the largest absolute change of $0.57^{[9]}$.

\section{Discussion}

Both discovered and undiscovered information is significant in this systematic review. With them, it is possible to confirm the results of existing studies as well as identify the future direction of research in this field, including the expansion and improvement of future studies.

It is obvious that adjusting the medical curriculum can improve the deficiencies of medical students and professionals in terms of knowledge and attitude. With appropriate supplementary knowledge, healthcare professionals will be more familiar with the protocol of LGBT health services and avoid making mistakes, such as incorrect pronouns. As more detailed information about LGBT patients is delivered, bias and discrimination can be mitigated as well.

However, further studies are required. In terms of the method to measure attitude change, self-reported pretests and post-tests had been employed in most studies. However, more studies should be designed through complex and reliable objective measurement. In addition, although single group and nonrandomized groups had been applied in existing research, stricter study designs such as randomized controlled trials should be implemented.

Moreover, most of the studies focus on medical students rather than clinical staffs, and the sample size is not large enough. Therefore, studies with continuing education and larger response rate are required. As for the outcomes of educational approaches, according to Kirkpatrick's level, researchers should not merely focus on knowledge and attitude; instead, further studies about participants' behavior and healthcare are required. Furthermore, in consideration of existing studies, most of them are conducted in the U.S.

\section{Conclusion}

This review documented the recurring concepts of LGBT in research into the curricula. As for the included studies in this review, the effectiveness of the LGBT curriculum has been revealed, although some processes, such as the outcome measurement and recruitment, can be improved. Due to the lack of evidence, it is impossible to compare the effect led by each method. Therefore, further trials and peer-reviewed reports, particularly high quality RCTs, are needed.

\section{Disclosure statement}

The author declares that there is no conflict of interest.

\section{References}

[1] Gates GJ, Newport F, 2012, Special Report: 3.4\% of US Adults Identified as LGBT, Gallup, Washington, DC.

[2] Noonan EJ, Sawning S, Combs R, et al., 2018, Engaging the Transgender Community to Improve Medical Education and Prioritize Healthcare Initiatives. Teaching and Learning in Medicine, 30(2): 119-132.

[3] Marsden J, 2014, Systematic Approaches to a Successful Literature Review. Nurse Researcher, 21(5): 46. 
[4] Grosz AM, Gutierrez D, Lui AA, et al., 2017, A Student-Led Introduction to Lesbian, Gay, Bisexual, and Transgender Health for First-Year Medical Students. Fam Med, 49(1): 52-56.

[5] Sawning S, Steinbock S, Croley R, et al., 2017, A First Step in Addressing Medical Education Curriculum Gaps in Lesbian-, Gay-, Bisexual-, And Transgender-Related Content: The University of Louisville Lesbian, Gay, Bisexual, and Transgender Health Certificate Program. Education for Health, 30(2): 108 .

[6] Safer JD, Pearce EN, 2013, A Simple Curriculum Content Change Increased Medical Student Comfort with Transgender Medicine. Endocrine Practice, 19(4): 633-637.

[7] White W, Brenman S, Paradis E, et al., 2015, Lesbian, Gay, Bisexual, and Transgender Patient Care: Medical Students' Preparedness and Comfort. Teaching and Learning in Medicine, 27(3): 254-263.

[8] Parameshwaran V, Cockbain BC, Hillyard M, et al., 2017, Is the Lack of Specific Lesbian, Gay, Bisexual, Transgender and Queer/Questioning (LGBTQ) Health Care Education in Medical School a Cause for Concern? Evidence from a Survey of Knowledge and Practice Among UK Medical Students. Journal of Homosexuality, 64(3): 367-381.

[9] Kelley L, Chou CL, Dibble SL, et al., 2008, A Critical Intervention in Lesbian, Gay, Bisexual, and Transgender Health: Knowledge and Attitude Outcomes Among Second-Year Medical Students. Teaching and Learning in Medicine, 20(3): 248-253. 